\title{
Elva Bañuelos Bárcena: una vida dedicada a la docencia de la economía
}

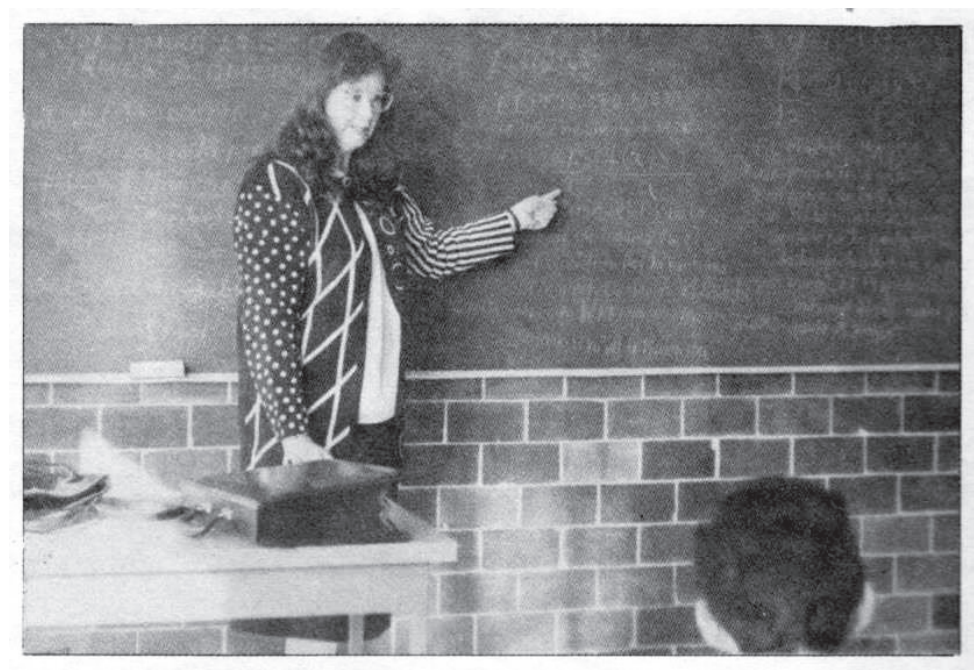

ecientemente galardonada con el Reconocimiento Sor Juana Inés de la Cruz (marzo de 2015), la profesora Elva Bañuelos Bárcena cuenta con una trayectoria docente de 46 años en la Facultad de Economía de la UNAM. Este breve artículo pretende ser un sencillo homenaje a la trayectoria docente de la profesora, quien ha dedicado los esfuerzos de toda una vida académica a la formación de profesionales de la ciencia económica en nuestro país.

\section{Formación académica}

Elva Bañuelos Bárcena nació en México, Distrito Federal. Cursó sus estudios de primaria en la escuela pública "Antonio García Cubas", después ingresó a la escuela secundaria Anexa a la Normal Superior de México, y sus estudios de preparatoria los realizó en la Escuela Nacional Preparatoria plantel número 1 "Gabino Barreda". Estudió la Licenciatura en Economía en la Escuela Nacional de Economía (hoy Facultad de Economía) de la Universidad Nacional Autónoma de México (UNAM) y se tituló en 1973 con la tesis "La dinámica del sector minero en México 1877-1970, con proyecciones a 1980”, obteniendo mención honorífica.

Más adelante, la profesora Bañuelos continuó capacitándose en diversos cursos de especialización. Durante 1995 y 1996 cursó el diplomado de Microeconomía organizado por la Dirección General de Asuntos del Personal Académico (DGAPA) de la UNAM, e integrado por cuatro módulos impartidos por destacados docentes de la UNAM (Dr. Fernando Noriega, Dra. Flor Brown y Dr. Felipe Peredo). Así mismo, entre 1996 y 1997, y en 2004, tomó tres cursos acerca del proceso enseñanza-aprendizaje. La profesora tiene la especialidad en "Mercados y Precios" y cursó un seminario de matemáticas impartido en la Facultad de Ciencias de la UNAM. 


\section{Ejercicio profesional en el sector público y privado}

La profesora Bañuelos también se desempeñó profesionalmente en el sector público de nuestro país. A la edad de 25 años, cuando tenía cubiertos los créditos académicos suficientes para ser "pasante" de la licenciatura en economía, ingresó a la Secretaria de Hacienda y Crédito Público (SHCP) como Asesor Analista en la Dirección de Estudios Hacendarios. En esa Dirección también tuvo actividad en los departamentos de Ingresos, Egresos y Deuda Pública.

Sus primeros trabajos en la SHCP correspondieron al sector de Egresos y Cuenta Pública, por lo que tuvo que estudiar previamente la metodología para la formulación de la Cuenta Pública. Más tarde participó en el Departamento de Ingresos, donde cursó varios seminarios, entre ellos, "Impuestos sobre la Renta", "Impuestos ad-valorem" y "Aplicación del IvA". También tomó un curso referente a la "Metodología para elaborar la Ley de Ingresos", lo que le permitió participar en la Comisión encargada de la elaboración de dicha Ley.

Posteriormente, la profesora Bañuelos pasó al Departamento de Deuda Pública donde realizó diversos trabajos sobre la deuda externa. En la sHCP, la profesora también participó en la Comisión que estaba encargada de informar al Banco de México sobre las Finanzas Públicas y preparaba la información que se incluía en los informes anuales del banco central.

Tras la experiencia en la SHCP, la profesora Bañuelos ingresó, en 1965, a la Secretaría de Industria y Comercio (hoy Secretaria de Economía), como Jefe del Departamento de Estudios Sectoriales y Regionales de la Dirección de Estudios Económicos. Las funciones que desempeñaba en ese puesto le permitieron conocer una faceta diferente del análisis de la actividad económica de nuestro país. En esa Secretaría de Estado, realizó diversos estudios sobre la industria harinera - en los cuales también participó personal de la Compañía Nacional de Subsistencias Populares (CONASUPO) - y sobre la industria del hierro y el acero en México. Así mismo, cursó un seminario sobre marcas y patentes en esa Dependencia.

La trayectoria de la profesora Bañuelos en la administración pública federal de nuestro país concluye con su participación, en 1968, en la Secretaria del Trabajo y Previsión Social (STPS), específicamente en la Dirección de Planeación Regional que tenía a su cargo la fijación de los salarios mínimos. En ese mismo año, la profesora incursionó en el sector privado al ingresar en la empresa FEMSA, en el Departamento de Alimentos y Bebidas, en la sección de Mercados, Publicidad, Ferias y Exposiciones. 


\section{Cargos académico-administrativos}

Dentro de los cargos académico-administrativos que ha ocupado la profesora Bañuelos en la Facultad de Economía y en la UNAM, se encuentran el de Coordinadora de la Academia de Teoría Económica (1987 a 1990), miembro del H. Consejo Técnico de la Facultad de Economía (1984 a 1987) y miembro del Claustro Académico para la Reforma del Estatuto del Personal Académico de la UNAM (2005 a 2010). Fue presidenta del Colegio de Profesores en la década de los ochenta y colaboradora de la Asociación Nacional de Instituciones de Docencia e Investigación Económica (ANIDIE) en la década de los noventa. Por su trayectoria académica, la profesora ha sido miembro del Jurado en tres concursos de oposición para ocupar plazas académicas en la Facultad de Economía de la UNAM.

\section{Una vida en la docencia: formadora de licenciados, profesores e investigadores}

La profesora Bañuelos inicia su brillante y larga trayectoria docente en 1969, al ser contratada por la entonces Escuela Nacional de Economía de la UNAM. Las primeras actividades docentes de la profesora consistieron en atender a los grupos que tomaban la asignatura que impartía el Centro de Economía Aplicada (CEA). Como parte de la capacitación requerida, el CEA envió a la profesora al Centro de Cálculo Electrónico para tomar los cursos de "Teoría de Gráficas y Redes", "Cibernética y Lenguajes de Computación” y "Métodos Matemáticos Aplicados a la Economía”.

En 1974 realizó, con éxito, su examen de oposición para optar por la plaza de Profesor Titular, de tiempo completo, definitivo, en el área de Teoría Económica, adscrita a la División de Estudios Profesionales. Así pues, en este 2015, la profesora Bañuelos cumple 46 años de trayectoria docente, de los cuales 41 han sido como profesora titular de tiempo completo impartiendo regularmente los cursos de microeconomía I y II, y macroeconomía I y II en la Facultad de Economía de la UNAM.

Una de las virtudes que caracterizan el trabajo docente de la profesora, es su constante actualización para estar a la vanguardia en los adelantos de la ciencia, en general, y de la economía, en particular. No ha habido libro nuevo relativo a la economía, que no haya leído la profesora, para luego comentar a sus estudiantes algunos pasajes, o incluso incorporar esos temas novedosos en sus clases. Esta constante actualización en el conocimiento de la ciencia económica 
le ha permitido a la profesora incluir en sus clases, a lo largo de cuatro décadas, numerosos ejemplos de problemas económicos actuales, ya sea de la economía mexicana o mundial.

En el campo metodológico, la profesora Bañuelos comenzó a trabajar en la elaboración de mapas conceptuales para la explicación de modelos económicos, mucho antes de que fueran una técnica docente generalizada en la Facultad de Economía de la UnAm. Así mismo, ha aplicado, desde hace más de dos décadas, el método constructivista para que los alumnos participen activamente en la construcción de los principales conceptos económicos.

Dentro de la obra de la profesora Bañuelos destacan los paquetes didácticos para las asignaturas de teoría microeconómica I y II, y los paquetes temáticos "Análisis sectorial de la economía mexicana", "Cápsulas económicas" y "Microeconomía conceptual". Durante el intenso proceso de cambio del Plan de Estudios de 1994, la profesora colaboró en la elaboración de las Cartas Descriptivas de las asignaturas microeconomía I, II, III y IV. La profesora también ha impartido cursos de capacitación, entre ellos, uno dirigido al personal del Banco de México, y otro para funcionarios del Ministerio de Precios de la República de Cuba.

Entre ensayos, tesinas y tesis, la profesora ha sido directora de un centenar de trabajos para titulación. Sin duda, hablamos de una de las docentes que más profesionales de la economía ha titulado en México.

Algunos de sus exalumnos ahora son famosos economistas con una brillante trayectoria profesional, como José Ángel Gurría Treviño, actual Secretario General de la Organización para la Cooperación y el Desarrollo Económico (OCDE). Otros de sus exalumnos ahora son sus colegas en la Facultad de Economía de la UNAM, como Jorge Méndez Hernández, de la generación 1979-1983, quien actualmente imparte cursos de teoría económica y recuerda a la profesora por su "profesionalismo en clases bien preparadas, fáciles de entender, y su casi nulo absentismo".

Entre los estudiantes más brillantes que han asistido a los cursos de la profesora está Abril Imelda Rosen Esquivel, de la generación 2001-2005, ganadora de la medalla al merito universitario Gabino Barreda por el mejor promedio (9.98), y quien se desempeñó como economista durante 9 años en el Banco de México, cursó en la Queen Mary University of London la maestría en Finanzas y Econometría, de la cual también se graduó con los más altos honores, y empezará en octubre de 2015 su doctorado en Finanzas en la Universidad de Essex gracias al apoyo de una beca del Economic and Social Research Council (ESRC) del Reino Unido. Abril Rosen opina de la profesora Elva Bañuelos: 
Además del carisma, sencillez y trato amable y responsable, es una persona sumamente profesional que muestra dominio de los temas y que además se distingue por su pedagogía, y por esa forma tan peculiar de hacer que lo difícil se torne tan sencillo.

Aún recuerdo los temas cuando curse Microeconomía II con la profesora: la competencia perfecta, la ineficiencia de los monopolios, los carteles de los oligopolios, los ejemplos prácticos de la Coca-Cola y la Pepsi-Cola, la importancia de la eficiencia paretiana y la teoría de juegos. Todos estos temas y muchos otros más fueron tan bien explicados por la profesora que aún permanecen en mi memoria. Puedo afirmar que todos estos conocimientos contribuyeron en gran medida para el éxito en mi examen de ingreso al Banco de México. Por todo esto y mucho más, siento un profundo agradecimiento hacia la profesora $\mathrm{Ba}-$ ñuelos, quien con su alto sentido moral y profesionalismo ha ayudado a formar economistas y a poner muy en alto el nombre de la UNAM.

Pero sus actividades de formación de capital humano no acaban ahí. La profesora Bañuelos ha sido una destacada formadora de profesores. Varios de aquellos jóvenes estudiantes que primero fueron sus alumnos y luego sus profesores adjuntos, ahora son docentes que imparten clases en diversas instituciones de educación superior del país, como Miguel Cervantes Jiménez, profesor titular en la Facultad de Economía de la UNAM, quien comenta:

Éramos estudiantes ávidos de conocimiento en un mar plagado de confusiones, sobre todo de orden ideológico. Cuando conocí a la profesora Elba Bañuelos, en el curso de microeconomía, ella ordenó la discusión en sus clases y me invitó a participar en sus grupos de iniciación temprana a la investigación. En una sesión de trabajo me dijo: "tienes que aprender el método de cada corriente de pensamiento económico y si quieres ser investigador, debes aprender que los problemas científicos se enfrentan sin prejuicios; los resultados de la investigación desvanecerán tus dudas".

La profesora Bañuelos, era y sigue siendo un pilar de la enseñanza de los dos enfoques de la teoría económica (micro y macroeconómico). Todos los exalumnos que le conozco, y que tengo el agrado de frecuentar, siempre se expresan con respeto, cariño y admiración hacia la profesora Bañuelos, no sólo por habernos enseñado el método de la teoría económica, sino también por habernos instruido valores, tales como la asiduidad, la puntualidad, el respeto y el valor para defender los ideales.

Siempre que he tenido la oportunidad le he agradecido el haberme incluido en sus equipos de investigación y haberme elegido como su profesor adjunto por varios años. Hoy la vida me vuelve a dar la oportunidad de decirle gracias 
por todo lo que le ha dado a la Facultad de Economía. Nuestro México requiere profesores como ella.

La profesora también ha destacado en la formación de jóvenes investigadores. Ha sido responsable de proyectos de investigación en dos programas específicos de la UNAM: el Programa de Iniciación Temprana a la Investigación y a la Docencia de Fundación UNAM, dentro del cual coordinó las investigaciones "La elasticidad y su importancia en el comercio exterior de México" y "El ahorro en México"; y el Programa de Becas para Tesis de Licenciatura en Proyectos de Investigación, en el cual dirigió la investigación "La estanflación de los países de la OCDE con aplicación de la curva de hillips". Uno de esos jóvenes investigadores fue Jorge Antonio Pérez Pineda, de la generación 1994-1998, quien actualmente se desempeña como profesor-investigador en el Instituto Mora y cuenta con el nivel I dentro del Sistema Nacional de Investigadores (SNI) del CONACYT. Jorge Pérez comenta:

$\mathrm{Su}$ orden metodológico plasmado en la pizarra, donde redactaba y nos presentaba el tema del día, nos permitía a su vez orden en las notas que tomábamos y facilitaba nuestro proceso enseñanza-aprendizaje de cara a las evaluaciones. También su incesante búsqueda por novedades editoriales que pudieran acercarnos materiales de calidad y primer nivel para el estudio de las materias que cursábamos con ella y para profundizar en todas las corrientes y variantes representativas que como economistas en formación debíamos cubrir. Destacaría también su cariño por impulsar jóvenes talentos de manera continua, y dando distintas oportunidades a aquellos que como yo se acercaron en su día para poder aprender más de nuestro oficio, bien fuera en el aula como adjunto, que enriquecía la clase, o en algún proyecto en la formación de jóvenes investigadores. Lo vivido con nuestra querida profesora me influiría trascendentalmente, pues eso me motivó a seguir preparándome y me llevo fuera del país a cursar estudios de posgrado, a vincularme a más investigaciones, y eventualmente a dedicarme a la academia. Creo que la generosidad intelectual de nuestra querida profesora fue siempre más allá de un profesor que cumplía con su horario y que sin duda ha dejado huella en nosotros y muchas generaciones que pasamos por sus cursos. Ojalá hubiera más profesores con esta vocación y amor no sólo por la economía, sino porque sus alumnos aprendan algo relevante y se formen.

En el campo de la difusión del conocimiento la profesora Bañuelos ha sido prolífica, pues ha participado en diversos eventos académicos con alrededor de 20 ponencias y 30 conferencias sobre temas de mercado, consumidores, empresas, impuestos, tecnología, telefonía y la riqueza de las naciones, entre otros. Algunas de sus conferencias han tenido eco en la prensa nacional, por ejemplo 
"Elección en el tiempo y el crédito a la vivienda"1 y "Discriminación de precios y la telefonía móvil en México". ${ }^{2}$ La profesora también ha difundido el conocimiento de la ciencia económica a través de artículos periodísticos, entre ellos “¿Qué sabe usted del consumo en México?”, "El ciclo de los negocios y el crédito bancario" y "El ahorro y el crédito social". 5

\section{Reconocimientos}

Entre los reconocimientos y distinciones que la UNAM ha entregado a la profesora Bañuelos, por su sobresaliente labor de docencia, se encuentran el Reconocimiento Sor Juana Inés de la Cruz, el Reconocimiento al Mérito Académico de la Asociación del Personal Académico de la UnAm, y el nivel "C" del Programa de Primas al Desempeño del Personal Académico de Tiempo Completo (PRI$\mathrm{DE})$.

El testimonio de la maestra Irma Escárcega Aguirre es muy elocuente acerca de los motivos por los cuáles son justos los reconocimientos recibidos por la profesora Elva Bañuelos:

A lo largo de mi vida adulta han quedado pendientes deudas insalvables, puede agradecerse, pero no hay manera alguna de pagar lo recibido. Cuando una institución como la UNAM hace posible un cambio de vida, abre caminos no conocidos, perspectivas no soñadas y proporciona conocimientos en todos los ámbitos, la deuda es infinita.

Y dentro de esta gran casa que nos acoge, sobresale la labor de los profesores que dejaron una muy particular huella en la formación personal. Elva Bañuelos forma parte de ese conjunto de maestros, que más allá del aula dejan con su presencia una impronta indeleble. Ha sido, para muchos como yo, un ejemplo por su paciencia dentro y fuera del salón de clase, su amable sonrisa, su personalidad calma, reflexiva, que a veces parece ingenua, su gesto sereno pero firme y su entrega total y absoluta a la labor docente. Por todo ello, justo ha sido reconocerla con el premio Sor Juana Inés de la Cruz a la docencia en la Facultad de Economía, que la gratitud de sus ex alumnos es permanente.

\footnotetext{
${ }^{1}$ Publicada por Notimex, "Aumentará la demanda de vivienda por pirámide poblacional en México”, en: http://www.prensamexicana.com/noticia/17109/aumentara-la-demanda-de-viviendapor-piramide-poblacional-en.

${ }^{2}$ Publicada por El Universal, “Utilizan celular más de 62 millones de mexicanos: unAm”, en: http:// www.eluniversal.com.mx/notas/459266.html.

${ }^{3}$ El Universal, 31 de enero de 2002.

${ }^{4}$ El Universal, 19 de abril de 2002.

${ }^{5}$ El Universal, 31 de mayo de 2002.
} 


\section{Una persona ejemplar}

No sólo en su trayectoria docente, sino también en su vida personal, la profesora Bañuelos es un ser humano ejemplar. El testimonio de sus hijas nos permite conocer, aunque sea de manera somera, algunas características de la mujer, de la madre, de la amiga, que comparte con gran amor las penas y las alegrías de la vida cotidiana con sus seres queridos:

Maribel: hablar de mi mamá es hablar de una gran mujer, de una hija amorosa y solidaria con sus padres y sus hermanos, buena amiga y compañera, ejemplo de entereza, de fortaleza, de honestidad y decisión para alcanzar las metas que se fue trazando con una visión de éxito. Como madre es un modelo a seguir, amorosa y protectora, enérgica y cálida. En los momentos más difíciles para sus hijos, un apoyo incondicional, basado en el amor que sólo una madre puede dar. Como docente es una gran formadora de profesionales, he sido testigo del esmero y la pasión por su trabajo, de su vocación, de su entrega y del sacrificio que exige su profesión. Es la persona que con su ejemplo llena mi vida de luz, en ella encuentro siempre el remanso que necesito, admiro su valentía, su inteligencia, su talento y capacidad de amar.

Marisol: estoy segura que si hubiera podido escoger a la madre más perfecta y brillante, la habría escogido a ella, dueña de toda mi admiración como profesionista y como ser humano. Siempre supo la ecuación perfecta: "más amor, multiplicado por disciplina, dividido entre su Universidad y su hogar, con un gran porcentaje de sabiduría, dando resultados precisos y perfectos". El mismo amor y esfuerzo que tuvo para criar a sus hijos lo tiene para preparar a generaciones de economistas, a veces hasta me ponía celosa del gran cariño y dedicación que mostraba por sus alumnos. Su gran valor agregado es el gran amor y entusiasmo con el que imparte sus conocimientos.

Humberto: Ahora que soy padre de un niño, valoro, entiendo y concientizo lo que significa mi mamá. Significa la fuerza, la perseverancia y el valor para sacar a tres niños adelante pese a la incertidumbre que nos da la vida día con día, no recuerdo un sólo momento en que nos haya fallado. Me siento orgulloso de que, además de guiarnos y apoyarnos incondicionalmente, haya tenido la capacidad de apoyar a cientos de jóvenes con sus conocimientos. Me produce una alegría infinita cuando se encuentra con algún ex alumno y la recuerdan con tanto cariño. Para mí, simplemente es la persona perfecta que Dios pudo mandar a mi vida. Le estaré eternamente agradecido, ya que gracias a ella mi vida está llena de alegrías y buenos momentos.Trataré de estar a su altura y seguir su ejemplo ahora con mi hijo. 


\section{Comentario final}

Con la profesora Elva Bañuelos cursé las materias de microeconomía y macroeconomía. Después participé con ella en el Programa de Iniciación Temprana a la Investigación y a la Docencia de Fundación unam, y en el Programa de Becas para Tesis de Licenciatura en Proyectos de Investigación, por lo que la profesora fue la directora de mi trabajo de tesis de licenciatura. Gracias a la profesora Bañuelos - y también a otros profesores como Aleida Guerrero Mondragón ${ }^{\dagger}$ y Aníbal Gutiérrez Lara- inicié mi carrera docente hace 20 años, colaborando como su profesor adjunto. Ya como colegas, mi relación con la profesora ha sido respetuosa y cercana.

No puedo concluir este artículo, sin antes mencionar que uno de los objetos que conservo con más afecto es una fotografía, publicada en la Gaceta de la Facultad de Economía en 1993, en la cual aparece la profesora Elva Bañuelos impartiendo clase, y en la parte inferior derecha de la imagen se alcanza a observar la nuca de un alumno sentado en la primera fila... ese alumno era yo. 